
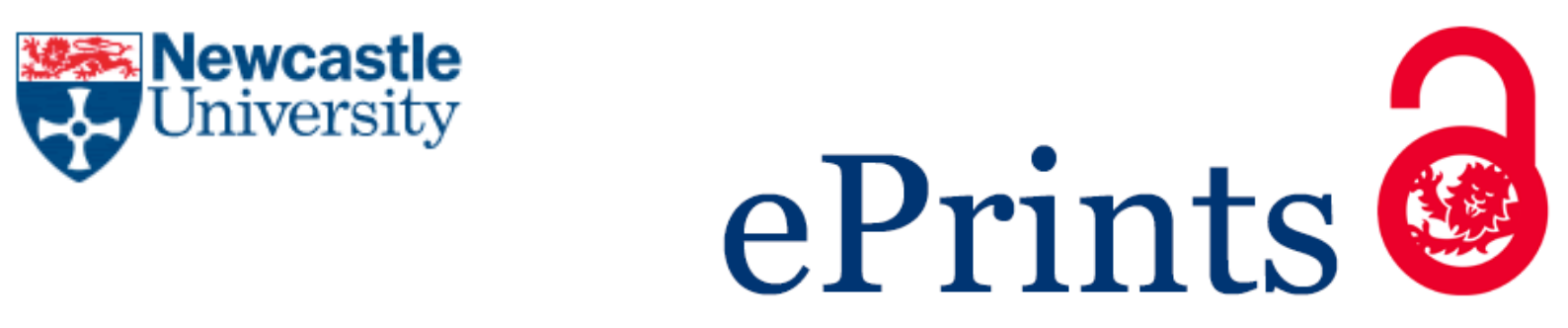

\author{
Papagiannidis S, Bourlakis M, Alamanos E, Dennis C. \\ Preferences of smart shopping channels and their impact on perceived \\ wellbeing and social inclusion. \\ Computers in Human Behaviour 2017 \\ DOI: https://doi.org/10.1016/j.chb.2017.04.029
}

Copyright:

(C) 2017. This manuscript version is made available under the CC-BY-NC-ND 4.0 license

DOI link to article:

https://doi.org/10.1016/i.chb.2017.04.029

Date deposited:

$11 / 05 / 2017$

Embargo release date:

12 April 2018

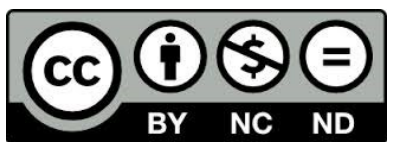

This work is licensed under a

Creative Commons Attribution-NonCommercial-NoDerivatives 4.0 International licence 


\section{Preferences of smart shopping channels and their impact on perceived}

wellbeing and social inclusion.

\section{Savvas Papagiannidis, Ph.D.}

David Goldman Professor of Innovation and Enterprise, Newcastle University Business School, 5 Barrack Road, Newcastle upon Tyne, NE1 4SE, UK.

Phone: +44 (0) 191208 1598; e-mail: savvas.papagiannidis@ncl.ac.uk

\section{Michael Bourlakis, Ph.D.}

Director of Demand Chain Management Community and Head of Supply Chain Research Centre, Cranfield School of Management, Cranfield, Bedford, MK43 0AL, UK. Phone: +44 (0) 123475 1122; e-mail: $\underline{\text { m.bourlakis@ @ cranfield.ac.uk }}$

\section{Eleftherios Alamanos*, Ph.D.}

Lecturer in Marketing, Newcastle University Business School, Newcastle University, Newcastle upon Tyne, NE1 4SE, UK.

Phone: +44 (0) 191208 1555; e-mail: Eleftherios.Alamanos@ newcastle.ac.uk

\section{Charles Dennis, Ph.D.}

Professor of Marketing and Retailing, The Business School, Middlesex University, London NW4 4BT, UK.

Phone: +44 (0) 208411 4463; e-mail: c.dennis@ mdx.ac.uk

* Corresponding author 


\title{
Preferences of smart shopping channels and their impact on perceived
}

\section{wellbeing and social inclusion.}

\begin{abstract}
This study examines consumers' interactions with retailers via three different shopping channels. Two of the channels are "smart" (technological) channels, comprising (i) where consumers shop using a computer and (ii) where consumers shop using a mobile phone. These two channels are compared with (iii) the traditional store channel. The paper explores the effect that consumers' interactions with these channels have on their wellbeing, with a focus on individuals who perceive themselves as being socially excluded, for example, lacking access to goods, services and information. We make a connection between social exclusion and channel contribution to wellbeing for multiple channels, through the lens of the Theory of Planned Behaviour. The online survey findings $(n=1368)$ indicate that for each channel, there is a higher contribution to wellbeing for that channel for people who are more socially excluded. Social exclusion can have many underlying causes, but channel contributions to wellbeing remain for consumers suffering financial stress and also those with mobility disability. For the mobile phone channel, the positive channel contributions to wellbeing are greater for younger than for older people. The paper outlines the implications for scholars and practitioners.
\end{abstract}

Keywords: multi-channel shopping, smart shopping channels, online shopping, social commerce, social exclusion, wellbeing. 


\section{Preferences of smart shopping channels and their impact on perceived wellbeing and social inclusion.}

\section{Introduction}

Interactions between consumers and innovative technologies that aim to enhance shopping experiences are often referred to as smart retailing, which is changing the way consumers access products, services and information (Blázquez, 2014; Pantano and Priporas, 2016; Pantano and Timmermans, 2014). Such purchase experiences can have important social benefits, can help to build shoppers' wellbeing and sometimes offset negative effects of social exclusion (Dennis, Alamanos, Papagiannidis and Bourlakis, 2015). This study examines consumers' interactions with retailers via three different shopping channels. Two of the channels are "smart" (technological) channels, comprising (i) where consumers shop using a computer and (ii) where consumers shop using a mobile phone. These two channels are compared with (iii) the traditional store channel. The paper explores the effect that consumers' interactions with these channels have on their wellbeing, with a focus on individuals who perceive themselves as being socially excluded, for example, lacking access to goods, services and information. Historically, shopping has made it possible for consumers to interact with others socially, which helped achieve integration and bring about a sense of community (Hewer and Campbell, 1997). With the advent of electronic channels, consumers found themselves in relative isolation from each other while undertaking their shopping online. Given the central role of shopping in our daily activities, it follows that the choice of retail channel can have an effect on how individuals interact, participate and integrate with their local communities. Consequently, understanding these choices and their impact can have significant implications.

Much academic effort has been invested in studying retail facilities in so-called 'excluded' and marginal neighbourhoods (Williams and Hubbard, 2001), for instance related to the closure of retail facilities in poorer areas and their relocation in more affluent ones (Guy, 1998; Westlake, 1993). A similar trend can be observed when consumers are the focus of attention, with studies examining disadvantaged individuals in deprived areas (Piacentini et al., 2001) or specific consumer segments 
(Hill, 2008). Still, given that social exclusion is a multidimensional construct that goes far beyond one's income, its manifestations can be widespread and permeate all parts of our societies. Consequently, there is a need to examine social exclusion using an ad hoc approach that does not embark from the assumption that living in a particular area or belonging to a certain group will result in a consumer being socially excluded. Instead, in this paper we recruited a consumer sample without imposing any restrictions such as the above, in order to examine the impact of social exclusion on the choices of retail channels consumers opt for. In turn, this current work studies the value they gain and how this contributes to their wellbeing. In doing so, this study aims to offer new, broader insights into social exclusion and the impact it can have on consumers, which could have significant practical implications for both retailers and policy makers.

The next section presents the conceptual model and associated hypotheses, before discussing the research design adopted. The results obtained from the structural equation modelling analysis are then presented and their implications discussed. The paper concludes by outlining the limitations of the paper and potential ways these limitations might be addressed in future projects.

\section{Literature Review}

Narrowly defined, social exclusion refers to income poverty either as a result of unemployment or low wages (Peace, 2001). Lack of financial resources can restrict access to goods, services and participation, which can have a negative effect on happiness and wellbeing (Taylor et al., 2011). Therefore, one could argue that people who are socially-included are more likely to be avid shoppers and have a positive attitude towards shopping. More broadly, though, social exclusion can refer to much more than poverty and income inequality. An individual who is geographically resident in a society is considered to be socially excluded if s/he cannot participate in the normal activities of citizens in that society, and s/he would like to participate, but is prevented from doing so by factors beyond their control (Burchardt et al., 1999). The factors that lead to social exclusion can vary from case to case depending on individual circumstances that have a significant impact on someone's wellbeing. Peace (2001) directly links social exclusion to well-being when he defines the former to be "the collective processes that work to deprive people of access to opportunities and means, material or otherwise, to achieve 
well-being and security in the terms that are important to them." For instance, beyond income-related factors that can lead to exclusion (Burchardt et al., 1999; Prawitz et al., 2006), this wider definition could potentially encompass a number of other dimensions that can also result in an individual being excluded, such as social area of residence, support networks, illness, age, family situation and mobility (Stanley et al., 2011; Wrigley et al., 2002; Piacentini et al., 2001).

This variability in factors might influence consumer preferences when it comes to selecting retail channels for their shopping needs. For instance, consumers who face mobility or disability challenges may experience difficulties when it comes to accessing stores and moving within them or when it comes to communicating with shop assistants (Swaine et al., 2014). Other individuals may be reluctant to visit stores due to psychological disorders such as agoraphobia (Belk, 2015). In such a case consumers may opt to use electronic channels, even though such a choice may result in their experience being less social. On the other hand, older consumers who tend to feel lonely and depressed when they have less social interaction (Kim et al., 2005) may do the opposite. Social exclusion may influence many factors related to retailing, but shopping can also potentially alleviate the consequences of social exclusion and not just help improve inclusion but also contribute to one's wellbeing and happiness. In order to study this process and the impact different channels have, we have adopted and adapted the Theory of Planned Behaviour (TPB). TPB suggests that attitudes, subjective norms and perceived behavioural control influence intentions and, in turn, behaviour. Personal attitudes towards a behaviour refer to the degree to which an individual has a favourable or unfavourable evaluation of that behaviour (Ajzen, 1991). Subjective norms describe the perceived social pressure to take a specific action. When consumers shop, they produce a self-image that others interpret (Sandikci and Holt, 1998) and hence they go through encounters that are constrained by the image they want others to have of them (Goffman, 1971 as cited by Baker, 2006). Perceived behavioural control is the perceived ease or difficulty of performing a given behaviour (Ajzen, 1991). The perceived ability of adopting a new behaviour can be influenced by both personality traits such as innovativeness and the level of involvement with a particular activity (Foxall, 1994). We have opted to use TPB for studying the psychological process related to selecting a channel as it has been found to successfully explain a wide range of human behaviours related to similar contexts 
to the one of this study, including traditional out-of-home (Carrington et al., 2014) and online (Hsu et al., 2006) shopping, and switching among offline and online channels (Pookulangara et al., 2011) as technology has infuenced consumption experiences (Zinkhan, 2005). Also, attitude, social norms and perceived behavioural control offer a parsimonious coverage of the effects that the underlying exclusion factors have. Based on the above we postulate that:

H1: Social exclusion negatively affects (a) the attitude, (b) the social norms and (c) the perceived behaviour control one has over a specific retail channel.

H2: (a) Attitude, (b) social norms and (c) perceived behaviour control positively affect the intentions to use a specific retail channel.

Shopping is not just about obtaining tangible products, but also enjoyment and socialising (Tauber, 1972). A rational selection would focus on maximising the value consumers get by shopping through a particular channel as well as the retailers' revenue as satisfactory shopping experiences can lead to a long-lasting relationship between a business and the customers (Walsh et al., 2016). For those who are socially excluded such decision making may involve factors that are beyond their control and hence such an attempt is inherently restricted from the outset. Consequently, this decision making process is not only a processing of maximising perceived value, but also one that potentially minimises the adverse effects of exclusion. Shopping online may bring lower prices yet lack personal interaction (Monsuwe et al., 2004), while visiting a mall may not be as efficient or convenient, but can result in a more enjoyable experience (Kim et al., 2005). Given that shopping can provide both utilitarian and hedonic value to consumers (Babin et al., 1994; Bellenger et al., 1977), the decision-making process can be seen as a balancing act between the utilitarian and hedonic value a customer gets when shopping via a specific channel. Utilitarian value is associated with the accomplishment of a task whilst hedonic value derives from fun or playfulness (Babin et al., 1994). For retailers, meeting shoppers` utilitarian values evokes satisfaction, whereas meeting hedonic expectations can evoke responses such as word of mouth recommendations (Chitturi et al., 2008). The main outcome for shoppers who have made a purchase is the hedonic value of accomplishing a task, whereas the main outcome for those who did not make a 
purchase is utilitarian values such as knowledge acquisition (Reynolds et al., 2012). Hedonic (Pookulangara et al., 2011) and utilitarian (Oppewal et al., 2013) beliefs influence channel-switching behaviour in traditional retailing and also in mobile retailing, where hedonic may have the stronger effect (Gao, Waechter, \& Bai, 2015; Kang, Mun, \& Johnson, 2015). Similarly, utilitarian beliefs influence attitude towards channel-switching in online (computer) retailing (Pookulangara et al., 2011). These arguments lead to:

H3: The intentions to use a retail channel positively affect the perceived (a) utilitarian and (b) hedonic value the consumer gets.

H4: The higher (a) the utilitarian and (b) the hedonic value a customer receives when shopping via a specific channel, the higher the contribution the channel makes to their wellbeing.

The above hypotheses 1-4 imply an indirect negative relationship between social exclusion and the channel contribution to wellbeing. Nevertheless, intuitively, a positive relationship is expected, due to the 'retail therapy' effect. Traditional mall shopping might help to alleviate the negative effects of social exclusion, increasing channel contribution to wellbeing (Dennis et al., 2007; Hedhli et al., 2013). Prior research similarly draws attention to the recreational and enjoyable aspects of online shopping (Field, 2005; Konus et al., 2008), which can also include peer-to-peer activities and transactions (Harris and Dumas, 2009). This can be of particular importance for socially-excluded consumers, for whom online shopping can be one of the main types of entertainment. Scholars argue that online shopping using a computer can help to alleviate the negative effects of social exclusion, increasing channel contribution to wellbeing (Dennis et al., 2007). In one empirical study, people who are lonely, socially isolated and living in poverty are given tablet computers, reporting, among many other benefits, a sense of connection with the outside world, keeping socially current, relaxing, improving mood and lifting depression (Irvine, 2016).

As the performance capabilities of mobile phones become more like those of computers (CalvoPorral \& Levy-Mangin, 2015), the benefits of e-shopping by computers should be available to and even enhanced by the experience of shopping by mobile phone (Pantano and Priporas, 2016). Mobile phone 
shopping should positively contribute to customers' channel contribution to wellbeing for two reasons. First, as the mobile phone accompanies the user whilst on the move, it can become almost an extension of the self and an integral part of socializing, for example: "iPhone is Facebook in my pocket... " (Harris and Dennis, 2011, p.342). Forty percent of mobile phone users access social networking sites via this device (Pew Internet, 2012). Second, the shopping value of the touchscreen interface of a smartphone can rival that of real products (Basel and Gips, 2014). These arguments lead to:

H5: The total effects of social exclusion on channel contribution to wellbeing are positive for channels (a) Mall, (b) Computer and (c) Mobile.

The above hypotheses are operationalised in the model depicted in Figure 1. As the model suggests, social exclusion influences a number of factors that affect the intention to use a specific retail channel. In turn, the channel choice affects the perceived utilitarian and hedonic value one gets while shopping, which make a contribution to the overall well-being. In addition, there is a direct, positive, link between social exclusion and channel contribution to wellbeing. The model is tested using a quantitative research design as discussed in the following section.

\section{Figure 1: Conceptual model}

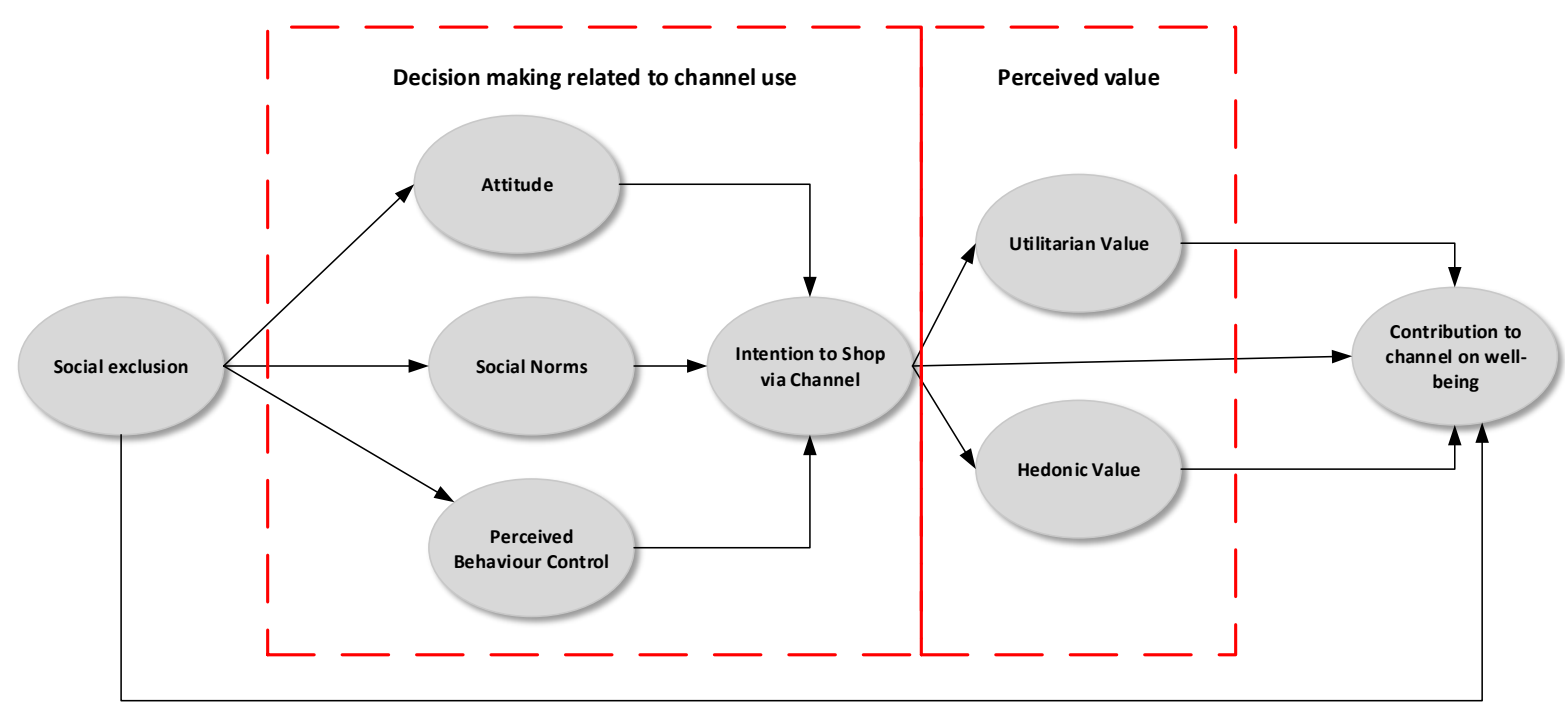


Social exclusion can have many underlying causes, including, for example, financial difficulties, mobility disabilities, remote geographical location and old age. Accordingly, the research design explores the extent to which the hypothesized relationships hold for shoppers: who are financially stressed compared with those who are not; those with mobility disabilities compared to those without disabilities; those with rural residence rather than urban and older as opposed to younger shoppers. In the interests of brevity, hypotheses are not developed for these but it may be that shopping, which demands financial resources, makes less contribution to wellbeing for people who are financiallystressed. Similarly, electronic channels may contribute less to wellbeing for older people, who may be less techno-literate.

\section{Methodology}

The data collection took place in the United States, the world's largest online market (Marketline, 2013). We recruited 1368 participants, aiming to balance the sample with regard to gender, age, and the participants' area of residence. Table 1 outlines our sample's characteristics. 
Table 1: Respondents' demographic and socioeconomic profile

\begin{tabular}{|c|c|c|c|c|c|}
\hline Characteristic & $\begin{array}{c}\text { Frequenc } \\
\mathbf{y}\end{array}$ & $\%$ & Characteristic & Frequency & $\%$ \\
\hline Gender & & & Financial Stress & & \\
\hline Male & 600 & $43.9 \%$ & Major Financial Stress & 492 & $36.0 \%$ \\
\hline Female & 768 & $56.1 \%$ & No / Minor Financial Stress & 876 & $64.0 \%$ \\
\hline Total & 1368 & $100.0 \%$ & Total & 1368 & $100.0 \%$ \\
\hline Age & & & Area of residence & & \\
\hline $20-39$ & 467 & $34.1 \%$ & Urbanised area & 476 & $34.8 \%$ \\
\hline $40-59$ & 464 & $33.9 \%$ & Urban cluster & 451 & $33.0 \%$ \\
\hline 60 or over & 437 & $31.9 \%$ & Rural & 441 & $32.2 \%$ \\
\hline Total & 1368 & $100.0 \%$ & Total & 1368 & $100.0 \%$ \\
\hline Employment Status & & & Educational attainment & & \\
\hline Full-time employed & 580 & $42.4 \%$ & Some high school or less & 7 & $0.5 \%$ \\
\hline Part-time employed & 169 & $12.4 \%$ & $\begin{array}{l}\text { High school graduate or } \\
\text { equivalent }\end{array}$ & 256 & $18.7 \%$ \\
\hline Out of work (looking for work) & 69 & $5.0 \%$ & $\begin{array}{l}\text { Vocational / technical } \\
\text { school (two year program) }\end{array}$ & 123 & $9.0 \%$ \\
\hline Out of work (not looking for work) & 11 & $0.8 \%$ & Some college but no degree & 331 & $24.2 \%$ \\
\hline Homemaker & 165 & $12.1 \%$ & $\begin{array}{l}\text { College graduate (four year } \\
\text { program) }\end{array}$ & 334 & $24.4 \%$ \\
\hline Student & 29 & $2.1 \%$ & Some graduate school & 69 & $5.0 \%$ \\
\hline Retired & 280 & $20.5 \%$ & Graduate degree & 205 & $15.0 \%$ \\
\hline Unable to work & 65 & $4.8 \%$ & Professional degree & 43 & $3.1 \%$ \\
\hline Total & 1368 & $100.0 \%$ & Total & 1368 & $100.0 \%$ \\
\hline Income & & & Disability and Mobility & & \\
\hline$\$ 0-\$ 24,999$ & 188 & $13.8 \%$ & $\begin{array}{l}\text { No / Minor disability and } \\
\text { mobility issues }\end{array}$ & 878 & $64.2 \%$ \\
\hline$\$ 25,000-\$ 49,999$ & 396 & $29.1 \%$ & & & \\
\hline$\$ 50,000-\$ 74,999$ & 344 & $25.2 \%$ & $\begin{array}{l}\text { Major disability and } \\
\text { mobility issues }\end{array}$ & 490 & $35.8 \%$ \\
\hline$\$ 75,000-\$ 99,9999$ & 234 & $17.2 \%$ & Total & 1368 & $100.0 \%$ \\
\hline More than $\$ 100,000$ & 201 & $14.7 \%$ & & & \\
\hline Total & 1363 & $100.0 \%$ & & & \\
\hline
\end{tabular}

In order to test our model's hypotheses for the chosen channels we adopted a number of previously validated scales to measure the variables in our models. We decided to study offline, out-of-home shopping, online via a personal computer and online shopping using mobile phones. Mobile phone shopping can be considered a distinct online channel offering features such as mobility and reachability (Wei et al., 2009). Consequently, as shopping online via a mobile phone can take place either in one's home or outside it could be considered an intermediate point between out-of-home and online shopping using a computer. Respondents answered on seven-point scales for all constructs (Table 2). Data collection took place over the Internet using an online questionnaire. The questions were presented to participants three times, once for each of the three channels considered, except for the social inclusion construct. 
Table 2: Measures

\begin{tabular}{|c|c|c|c|c|}
\hline \multirow{2}{*}{ Construct } & \multirow{2}{*}{ Source } & \multicolumn{3}{|c|}{ Loading } \\
\hline & & Computer & $\begin{array}{l}\text { Mobile } \\
\text { Phone }\end{array}$ & Mall \\
\hline \multicolumn{2}{|l|}{ Hedonic Value } & C.R. $=.947$ & C.R. $=.984$ & C.R. $=.966$ \\
\hline Shopping truly feels like an escape. & \multirow{5}{*}{ (Babin et al., 1994) } & .882 & .951 & .919 \\
\hline $\begin{array}{l}\text { While shopping, I had a good time because I was } \\
\text { able to act on the "spur-of-the-moment. " " }\end{array}$ & & .883 & .965 & .916 \\
\hline $\begin{array}{l}\text { I enjoy shopping for its own sake, not just for the } \\
\text { items I may purchase. }\end{array}$ & & .873 & .961 & .923 \\
\hline $\begin{array}{l}\text { During a given shopping session, I felt the } \\
\text { excitement of the hunt. }\end{array}$ & & .898 & .964 & .933 \\
\hline While shopping, I felt a sense of adventure. & & .884 & .962 & .921 \\
\hline \multicolumn{2}{|l|}{ Utilitarian Value } & C.R. $=.840$ & C.R. $=.956$ & C.R. $=.900$ \\
\hline $\begin{array}{l}\text { On a given shopping session, I accomplished just } \\
\text { what I wanted. }\end{array}$ & \multirow{2}{*}{ (Babin et al., 1994) } & .868 & .966 & .933 \\
\hline $\begin{array}{l}\text { On a given shopping session, I found just the } \\
\text { item(s) I was looking for. }\end{array}$ & & .834 & .948 & .875 \\
\hline \multicolumn{2}{|l|}{ Subjective Norms } & C.R. $=.922$ & C.R. $=.967$ & C.R. $=.952$ \\
\hline $\begin{array}{l}\text { People who are important to me think that I should } \\
\text { shop. }\end{array}$ & \multirow{3}{*}{ (Yang, 2012) } & .923 & .950 & .943 \\
\hline $\begin{array}{l}\text { I would shop because of the proportion of my } \\
\text { friends who shop online using a computer. }\end{array}$ & & .906 & .960 & .921 \\
\hline $\begin{array}{l}\text { People who influence my behaviour think that I } \\
\text { should shop. }\end{array}$ & & .848 & .948 & .933 \\
\hline \multicolumn{2}{|l|}{ Perceived Behavioural Control } & C.R. $=.854$ & C.R. $=.862$ & C.R. $=.843$ \\
\hline I have access to shopping. & \multirow[b]{2}{*}{ (Yang, 2012) } & .797 & .786 & .776 \\
\hline $\begin{array}{l}\text { Given the resources, opportunities and knowledge } \\
\text { it takes to shop, it would be easy for me to shop. }\end{array}$ & & .926 & .949 & .927 \\
\hline \multicolumn{2}{|l|}{ Attitude } & C.R. $=.918$ & C.R. $=.953$ & C.R. $=.924$ \\
\hline I have a positive opinion about shopping. & \multirow{2}{*}{ (Hsu et al., 2006) } & .912 & .953 & .922 \\
\hline Shopping is appropriate for me. & & .918 & .966 & .928 \\
\hline Shopping is a good idea. & (Yang, 2012) & .831 & .878 & .834 \\
\hline \multicolumn{2}{|l|}{ Social Exclusion (asked once) } & C.R. $=.947$ & C.R. $=.947$ & C.R. $=.947$ \\
\hline I do not have access to goods and services. & (Huxley et al., 2012) & .816 & .816 & .816 \\
\hline There is no one I can turn to if I need support. & \multirow{3}{*}{$\begin{array}{l}\text { (Liu and Forsythe, } \\
\text { 2011) }\end{array}$} & .951 & .951 & .951 \\
\hline I feel left out. & & .931 & .931 & .931 \\
\hline I lack companionship. & & .913 & .912 & .913 \\
\hline Channel Contribution to Wellbeing & & C.R. $=.943$ & C.R. $=.985$ & C.R. $=.963$ \\
\hline $\begin{array}{l}\text { Shopping plays a very important role in my social } \\
\text { well-being. }\end{array}$ & \multirow{3}{*}{ (Hedhli et al., 2013) } & .932 & .978 & .966 \\
\hline $\begin{array}{l}\text { Shopping plays a very important role in my leisure } \\
\text { well-being. }\end{array}$ & & .926 & .980 & .960 \\
\hline $\begin{array}{l}\text { Shopping plays an important role in enhancing the } \\
\text { quality of my life in my community. }\end{array}$ & & .902 & .975 & .914 \\
\hline Intention & & C.R. $=.929$ & C.R. $=.979$ & C.R. $=.958$ \\
\hline Given the chance, I intend to shop. & \multirow{3}{*}{ (Yang, 2012) } & .846 & .968 & .915 \\
\hline I expect my shopping to continue in the future. & & .939 & .967 & .956 \\
\hline I intend to purchase products or services. & & .919 & .973 & .949 \\
\hline
\end{tabular}

Notes: CR: Construct Reliability: is computed from the sum of factor loadings ( $\lambda i$ ), squared for each construct and the sum of the error variance terms for a construct ( $\delta i)$ using the formula below. A CR estimate $\geq .7$ suggests good reliability (Hair et al., 2010).

$$
C R=\frac{\left(\sum_{i=1}^{n} \lambda_{i}\right)^{2}}{\left(\sum_{i=1}^{n} \lambda_{i}\right)^{2}+\left(\sum_{i=1}^{n} \delta_{i}\right)}
$$




\section{Results}

Structural equation modelling examined the relationships between the concepts that influence shopping intentions, either via traditional retailing (shopping at the mall, or online via computer or mobile phone), and the effect that this has on shoppers'wellbeing. We ran the analysis separately for the three channels. Discriminant and convergent validity were satisfactory (Table 3).

\section{Table 3: Discriminant validity and average variance explained}

\begin{tabular}{|c|c|c|c|c|c|c|c|c|c|}
\hline \multicolumn{10}{|c|}{ Computer } \\
\hline Construct & AVE & 1 & 2 & 3 & 4 & 5 & 6 & 7 & 8 \\
\hline 1 Perceived Behaviour Control & 0.746 & 0.864 & & & & & & & \\
\hline 2 Hedonic Motivations & 0.782 & 0.384 & 0.884 & & & & & & \\
\hline 3 Utilitarian Motivations & 0.724 & 0.758 & 0.556 & 0.851 & & & & & \\
\hline 4 Social Exclusion & 0.818 & -0.182 & 0.226 & -0.036 & 0.904 & & & & \\
\hline 5 Attitude & 0.788 & 0.829 & 0.498 & 0.802 & -0.144 & 0.888 & & & \\
\hline 6 Well-being & 0.847 & 0.213 & 0.849 & 0.420 & 0.344 & 0.354 & 0.920 & & \\
\hline 7 Social Norms & 0.797 & 0.198 & 0.696 & 0.345 & 0.357 & 0.294 & 0.762 & 0.893 & \\
\hline 8 Intentions & 0.814 & 0.824 & 0.456 & 0.832 & -0.150 & 0.865 & 0.286 & 0.208 & 0.902 \\
\hline \multicolumn{10}{|c|}{ Mobile Phones } \\
\hline Construct & AVE & 1 & 2 & 3 & 4 & 5 & 6 & 7 & 8 \\
\hline 1 Perceived Behaviour Control & 0.759 & 0.871 & & & & & & & \\
\hline 2 Hedonic Motivations & 0.923 & 0.777 & 0.961 & & & & & & \\
\hline 3 Utilitarian Motivations & 0.916 & 0.827 & 0.945 & 0.957 & & & & & \\
\hline 4 Social Exclusion & 0.817 & 0.286 & 0.450 & 0.411 & 0.904 & & & & \\
\hline 5 Attitude & 0.871 & 0.834 & 0.922 & 0.917 & 0.391 & 0.933 & & & \\
\hline 6 Well-being & 0.956 & 0.720 & 0.944 & 0.883 & 0.487 & 0.872 & 0.978 & & \\
\hline 7 Social Norms & 0.908 & 0.741 & 0.922 & 0.868 & 0.483 & 0.890 & 0.924 & 0.953 & \\
\hline 8 Intentions & 0.940 & 0.798 & 0.936 & 0.938 & 0.414 & 0.932 & 0.910 & 0.878 & 0.969 \\
\hline \multicolumn{10}{|c|}{ Out-of Home } \\
\hline Construct & AVE & 1 & 2 & 3 & 4 & 5 & 6 & 7 & 8 \\
\hline 1 Perceived Behaviour Control & 0.731 & 0.855 & & & & & & & \\
\hline 2 Hedonic Motivations & 0.851 & 0.586 & 0.922 & & & & & & \\
\hline 3 Utilitarian Motivations & 0.818 & 0.735 & 0.715 & 0.904 & & & & & \\
\hline 4 Social Exclusion & 0.818 & -0.053 & 0.187 & 0.064 & 0.904 & & & & \\
\hline 5 Attitude & 0.802 & 0.781 & 0.800 & 0.787 & 0.049 & 0.896 & & & \\
\hline 6 Well-being & 0.897 & 0.472 & 0.858 & 0.619 & 0.258 & 0.684 & 0.947 & & \\
\hline 7 Social Norms & 0.869 & 0.418 & 0.717 & 0.568 & 0.303 & 0.589 & 0.798 & 0.932 & \\
\hline 8 Intentions & 0.884 & 0.774 & 0.751 & 0.836 & 0.024 & 0.883 & 0.621 & 0.524 & 0.940 \\
\hline
\end{tabular}

Notes:

${ }^{1}$ AVE: Average Variance Explained. AVE should be $\geq .5$ to suggest adequate Convergent Validity.

${ }^{2}$ The diagonal of the table presents the square root of AVE. Numbers below the diagonal represent the correlations between the factors. The square root of the AVE estimates should be greater than the correlations between that factor and other factors to provide evidence of Discriminant Validity (Hair et al., 2010). 
Results for the three models (Table 4) indicate a strong fit. All items load significantly under their respective factors, demonstrating a good reliability of the scales (Hair et al., 2010).

Table 4: Structural equation models

\begin{tabular}{|c|c|c|c|c|c|c|}
\hline \multirow[b]{2}{*}{ Path } & \multicolumn{2}{|c|}{ Computer } & \multicolumn{2}{|c|}{ Mobile Phone } & \multicolumn{2}{|c|}{ Mall } \\
\hline & $\begin{array}{c}\text { Standardised } \\
\text { Coefficient }\end{array}$ & t-test & $\begin{array}{c}\text { Standardised } \\
\text { Coefficient }\end{array}$ & t-test & $\begin{array}{c}\text { Standardised } \\
\text { Coefficient }\end{array}$ & t-test \\
\hline $\begin{array}{l}\text { Social Exclusion } \rightarrow \text { Social } \\
\text { Norms }\end{array}$ & .357 & $12.776^{* * *}$ & .483 & $18.748^{* * *}$ & .303 & $11.026 * * *$ \\
\hline Social Exclusion $\rightarrow$ PBC & -.289 & $-9.490 * * *$ & -.094 & $-3.923 * * *$ & -.197 & $-6.982 * * *$ \\
\hline Social Norms $\rightarrow$ PBC & .301 & $9.661 * * *$ & .786 & $31.762 * * *$ & .478 & $16.649 * * *$ \\
\hline Social Exclusion $\rightarrow$ Attitude & -.058 & $-2.733 * *$ & -.014 & $-.935 \mathrm{~ns}$ & -.014 & $-.723 \mathrm{~ns}$ \\
\hline $\mathrm{PBC} \rightarrow$ Attitude & .787 & $30.857 * * *$ & .386 & $16.392 * * *$ & .645 & $24.537 * * *$ \\
\hline Social Norms $\rightarrow$ Attitude & .159 & $7.276^{* * * *}$ & .610 & $25.044 * * *$ & .323 & $13.936^{* * * *}$ \\
\hline Social Exclusion $\rightarrow$ Intentions & .012 & $.648 \mathrm{~ns}$ & .028 & $2.187 *$ & -.005 & $-.343 \mathrm{~ns}$ \\
\hline PBC $\rightarrow$ Intentions & .338 & $8.967 * * *$ & .072 & $3.073 * *$ & .217 & $7.303 * * *$ \\
\hline Social Norms $\rightarrow$ Intentions & -.039 & $-2.052 *$ & .216 & $7.465 * * *$ & .022 & $1.087 \mathrm{~ns}$ \\
\hline Attitude $\rightarrow$ Intentions & .598 & $15.431 * * *$ & .668 & $18.603 * * *$ & .701 & $20.814 * * *$ \\
\hline Social Exclusion $\rightarrow$ Utilit. Value & .051 & $2.358 *$ & .019 & $1.530 \mathrm{~ns}$ & .009 & $.514 \mathrm{~ns}$ \\
\hline PBC $\rightarrow$ Utilit. Value & .153 & $3.335 * * *$ & .168 & $7.130 * * *$ & .208 & $5.943 * * *$ \\
\hline Social Norms $\rightarrow$ Utilit. Value & .131 & $5.702 * * *$ & .108 & $3.820 * * *$ & .166 & $7.067 * * *$ \\
\hline Attitude $\rightarrow$ Utilit. Value & .199 & $3.772 * * *$ & .151 & $3.504 * * *$ & .031 & $.628 \mathrm{~ns}$ \\
\hline Intentions $\rightarrow$ Utilit. Value & .514 & $10.154 * * *$ & .561 & $15.773 * * *$ & .560 & $12.384 * * *$ \\
\hline Social Exclusion $\rightarrow$ Hed. Value & .057 & $2.579 * *$ & .012 & $1.205 \mathrm{~ns}$ & .046 & $2.761 * *$ \\
\hline $\mathrm{PBC} \rightarrow$ Hedonic Value & -.115 & $-2.442 *$ & -.076 & $-4.036 * * *$ & -.123 & $-3.822 * * *$ \\
\hline Social Norms $\rightarrow$ Hed. Value & .549 & $20.445^{* * *}$ & .326 & $14.634 * * *$ & .332 & $14.762 * * *$ \\
\hline Attitude $\rightarrow$ Hed. Value & .137 & $2.526^{*}$ & .111 & $3.341 * * *$ & .456 & $10.014 * * *$ \\
\hline Intentions $\rightarrow$ Hed. Value & .127 & $2.209 *$ & .186 & $5.503 * * *$ & .183 & $4.023 * * *$ \\
\hline Utilit. Value $\rightarrow$ Hed. Value & .241 & $4.684 * * *$ & .445 & $12.884 * * *$ & .103 & $2.863 * *$ \\
\hline Social Exclusion $\rightarrow$ Wellbeing & .078 & $4.485 * * *$ & .042 & $3.949 * * *$ & .032 & $2.131 *$ \\
\hline $\mathrm{PBC} \rightarrow$ Wellbeing & -.138 & $-3.676 * * *$ & -.026 & $-1.248 \mathrm{~ns}$ & -.047 & $-1.619 \mathrm{~ns}$ \\
\hline Social Norms $\rightarrow$ Wellbeing & .284 & $11.358 * * *$ & .345 & $11.882 * * *$ & .363 & $16.188 * * *$ \\
\hline Attitude $\rightarrow$ Wellbeing & .082 & $1.911 \#$ & -.167 & $-4.514 * * *$ & .050 & $1.166 \mathrm{~ns}$ \\
\hline Intentions $\rightarrow$ Wellbeing & -.053 & $-1.186 n s$ & .354 & $9.501 * * *$ & -.030 & $-.747 \mathrm{~ns}$ \\
\hline Hed. Value $\rightarrow$ Wellbeing & .642 & $22.278 * * *$ & .596 & $12.849 * * *$ & .601 & $19.227 * * *$ \\
\hline Utilit. Value $\rightarrow$ Wellbeing & .051 & $1.256 \mathrm{~ns}$ & -.154 & $-3.473 * * *$ & .000 & $.013 \mathrm{~ns}$ \\
\hline
\end{tabular}

Notes: Model Fit

Mall: Method: $M L ;$ Model fit: $\chi 2(247)=1157.838, C M I N / D F=4.688, C F I=.979$, RMSEA $=.052$

Computer: Method: $M L ;$ Model fit: $\chi 2(247)=1066.785, C M I N / D F=4.319, C F I=.977, R M S E A=.049$

Mobile Phone: Method: ML; Model fit: $\chi 2(247)=1023.298, C M I N / D F=4.143, C F I=.987, R M S E A=.048$

Significant at $p: n s \geq .1 ; \# \leq .1 ; * \leq .05 ; * * \leq .01 ; * * * \leq .001$

\subsection{Shopping online using a computer}

In the case of online shopping using a computer, social exclusion has a positive effect on the importance of social norms when considering shopping via this channel (H1b rejected). However, it has a negative effect on perceived behavioural control (H1c) and with these findings illustrating possible social pressure and unfavourable views when using this channel. In contrast, social norms have a positive effect on perceived behavioural control. Perceived social exclusion also has a negative effect on respondents' attitude towards shopping online using a computer (H1a) suggesting the negative 
sentiments created by social exclusion in connection with this channel. In contrast, perceived behavioural control and social norms have a positive effect on attitude towards shopping online using a computer. Perceived behavioural control $(\mathrm{H} 2 \mathrm{c})$ and attitude (H2a) towards online shopping using a computer also have a positive effect on intentions towards using this shopping channel; these findings show the clear contribution of both perceived behaviour control and attitude towards specific behavioural traits (intentions) when using the computer. In contrast, the effect of social norms on intentions is negative ( $\mathrm{H} 2 \mathrm{~b}$ rejected) and there was no effect of social exclusion on shopping online using a computer. Social exclusion, attitude, social norms, and intentions of shopping online using a computer have a positive effect on the perceived utilitarian and hedonic value from shopping via this channel ( $\mathrm{H} 3 \mathrm{a}$ and $\mathrm{H} 3 \mathrm{~b})$ indicating the critical role of this channel for generating core / fundamental values to these consumers. Perceived behavioural control of shopping online via a computer has a positive effect on the perceived utilitarian value, but it has a negative effect on the perceived hedonic value of shopping online using a computer. The perceived utilitarian value also enhances the perceived hedonic value of shopping online using a computer and the latter suggests possible synergies and interrelationships between both sets of values (utilitarian and hedonic). In addition, the more socially excluded an individual feels the higher the perceived contribution of shopping online via a computer on this person's wellbeing is. Social norms and hedonic value also have a significant positive effect on the contribution of this shopping channel on an individual's wellbeing. These are key findings illustrating the major role of these issues towards individual wellbeing. The perceived behavioural control of shopping online using a computer has a negative effect on the perceived contribution of the channel on wellbeing. Finally, the perceived utilitarian value of shopping online using a computer has a nonsignificant effect on wellbeing (H4a rejected) whereas the perceived hedonic value of shopping online using a computer has a positive effect on wellbeing $(\mathrm{H} 4 \mathrm{~b})$; the latter could be attributed to the possible creation of positive emotions via shopping online which, in turn, could create a positive influence towards wellbeing. 


\subsection{Shopping online using a mobile phone}

Social exclusion also has a positive effect on the importance of social norms when considering shopping online using a mobile phone ( $\mathrm{H} 1 \mathrm{~b}$ rejected). In contrast, the effect of social exclusion on the perceived behavioural control of shopping via this channel was negative (H1c) whereas there was no effect of social exclusion on attitude towards shopping via a mobile phone (H1a rejected). These findings provide an insightful perspective for the role of social exclusion towards specific behavioural aspects when shopping with a mobile phone. Social norms have a positive effect on the perceived behavioural control of shopping via this channel (H1a rejected). Social norms and perceived behavioural control also have a positive effect on attitude towards shopping online using a mobile phone. Social exclusion, attitude (H2a), social norms (H2b) and perceived behavioural control (H2c) also positively influence the intentions of shopping online via a mobile phone. Hence, these findings suggest a clear interrelationship of factors influencing consumers' intention to shop online with a mobile phone. The degree of an individual's social exclusion does not have an effect on the perceived utilitarian value of shopping online via a mobile phone. In contrast, attitude, social norms, perceived behavioural control and intentions (H3a) all have positive effects on the perceived utilitarian value of shopping online via a mobile phone indicating the contribution of these traits towards the creation of an "utility" when using this channel. Feeling socially excluded does not have an effect on the perceived hedonic value from shopping online using a mobile phone whereas perceived behavioural control negatively influences the hedonic value. In contrast, social norms, attitude, intentions (H3b) and the perceived utilitarian value positively influence the perceived hedonic value which highlights the synergies and interrelationships between these issues. Being socially excluded positively influences the perceived contribution of shopping online via a mobile phone on an individual's wellbeing. In contrast, perceived behavioural control does not influence wellbeing which is a different result compared to what we noted when using a computer. Social norms have a positive effect on the perceived contribution of the channel on an individual's wellbeing. In contrast, the effect of attitude towards shopping online using a mobile has a negative effect on the perceived contribution of the channel on an individual's wellbeing. The effect of intentions of shopping online using a mobile phone and of the anticipated hedonic value positively influence the perceived contribution of the channel on an individual's wellbeing. Finally, the 
perceived utilitarian value of shopping online using a mobile phone has a negative effect on wellbeing (H4a rejected) whereas the perceived hedonic value of shopping online using a mobile phone has a positive effect on wellbeing (H4b). Overall, the above findings delineate clear similarities and differences for the role of the computer and the mobile phone when shopping online as some of these hypotheses had different outcomes for the two channels involved.

\subsection{Shopping at the mall}

In relation to shopping at the mall, feeling socially excluded has a positive effect on the importance of social norms in relation to the decision of shopping via this channel (H1b rejected). This is not a surprising result as socially excluded consumers may feel pressured to socialise and end up going to the mall. Social exclusion has a negative effect on the perceived behavioural control of shopping at the mall (H1c) whereas social norms have a positive effect on perceived behavioural control; the latter indicates the contrasting influence of these issues towards perceived behavioural control. Social exclusion does not have an effect on attitude towards shopping at the mall (H1a rejected) whereas social norms and perceived behavioural control positively influence attitude towards shopping at the mall. Social exclusion and social norms do not have an effect on intentions to shop at the mall. In contrast, attitude (H2a) and perceived behavioural control (H2c) positively influence intentions to shop at the mall and similar findings were noted for the other two channels too. Neither do social exclusion and social norms affect intentions to shop at the mall ( $\mathrm{H} 2 \mathrm{~b}$ rejected). The perceived utilitarian value of shopping at the mall is not influenced by social exclusion and attitude whereas it is positively affected by social norms, perceived behavioural control and intentions (H3a). The perceived hedonic value of shopping at the mall is influenced by social exclusion, social norms, attitude and intentions (H3b), as consumers may perceive positively the enjoyment being the mall and its social contribution to their lives) whereas it is negatively affected by perceived behavioural control as consumers may perceive getting utility / value by other channels too. In addition, feeling socially excluded has a positive effect on the perceived contribution of shopping at the mall to an individual 's wellbeing and this is a major finding. In contrast, perceived behavioural control does not affect wellbeing. Social norms and the anticipated hedonic value (H4b) from shopping at the mall positively influence the perceived contribution of the channel to an 
individual's wellbeing and a similar finding was noted for the other two channels. In contrast, attitude, intentions and the perceived utilitarian value do not have such an effect (H4a rejected). The standardised total effects of social exclusion on channel contribution to wellbeing are positive for each channel: mall $.258(\mathrm{H} 5 \mathrm{a})$, computer $.344(\mathrm{H} 5 \mathrm{~b})$ and the highest is for the mobile phone $.461(\mathrm{H} 5 \mathrm{c})$.

\subsection{Moderating Variables}

To evaluate the possible influences of moderating variables such as gender, age, time spent shopping, financial stress, disability/mobility and rural/urban residence, multi-group analyses were performed in SPSS Amos (although details are omitted for brevity). There were few significant differences in path weights between the groups, although, for example, three paths (subjective norm $\rightarrow$ intention, social exclusion $\rightarrow$ utilitarian value and social exclusion $\rightarrow$ channel contribution to wellbeing) were weaker for the mobile phone channel for respondents reporting severe financial stress than for those with low financial stress. Similarly, four paths (social exclusion $\rightarrow$ perceived behavioural control, social exclusion $\rightarrow$ attitude, perceived behavioural control $\rightarrow$ channel contribution to wellbeing (negative path), and attitude $\rightarrow$ channel contribution to wellbeing (negative path)) were weaker for the mobile phone channel for respondents reporting major disability issues than for those with no disability issues. Six paths (social exclusion $\rightarrow$ subjective norm, perceived behavioural control $\rightarrow$ attitude, subjective norm $\rightarrow$ hedonic value, intention $\rightarrow$ hedonic value, social exclusion $\rightarrow$ channel contribution to wellbeing, and hedonic value $\rightarrow$ channel contribution to wellbeing) were weaker for the mobile phone channel for older compared to younger respondents.

Notwithstanding various small differences such as these, the total effects of social exclusion on channel contribution to wellbeing are positive for each channel and every moderating variable (H5a, b and c confirmed for each moderating variable). In every case, for each channel, there is a higher contribution to wellbeing for that channel for people who are more socially excluded. The only significant differences across the moderating variables are that for the mobile phone: the effects are greater for those with low financial stress than for those suffering severe financial stress; and for the mobile phone and computer: the effects are greater for younger than for older people (Table 5). 
Table 5: Standardised total effects of social exclusion on channel contribution to wellbeing for moderators with significant differences for that direct path.

\begin{tabular}{|l|c|c|c|c|}
\hline & $\mathbf{\Delta} \boldsymbol{\chi}^{\mathbf{2}}$ & Sig & $\begin{array}{c}\text { Low financial stress } \\
\text { Standardised Coefficient }\end{array}$ & $\begin{array}{c}\text { Severe financial stress } \\
\text { Standardised Coefficient }\end{array}$ \\
\hline Financial stress & & & .555 & .358 \\
\hline mobile phone & 4.79 & $*$ & .375 & .280 \\
\hline Computer & .012 & $\mathrm{~ns}$ & .983 & .436 \\
\hline Mall & .226 & $\mathrm{~ns}$ & $\mathbf{2 0 - 4 9}$ & $\mathbf{5 0}$ and over \\
\hline Age & & & Standardised Coefficient & Standardised Coefficient \\
\hline mobile phone & 9.70 & $* *$ & .506 & .312 \\
\hline Computer & 4.42 & $*$ & .306 & .275 \\
\hline
\end{tabular}

Notes:

${ }^{1}$ Partial metric invariance was not established for moderator age for the mall channel. There are no significant differences for the effect of social exclusion on channel contribution to wellbeing for the other moderators studied.

${ }^{2}$ The statistical significance of the differences between the standardised coefficients for each pair of groups (i.e. Low financial stress versus Severe Financial Stress and 20-49 versus 50 and over) was tested by examining the statistical significance of the difference in the value of $\chi^{2}$ test of the respective structural equation models following the establishment of partial metric invariance between them.

\section{Discussion}

The findings suggest that social exclusion has a positive effect on subjective norms via all channels. In contrast, social exclusion has a negative effect on perceived behavioural control via all channels. Hence the more socially excluded an individual feels, the more confident they feel in shopping via each channel. Subjective norms also have a positive effect on perceived behavioural control. Hence perceived behavioural control can be enhanced by peers' influences. These findings shed light on the use of TPB in relation to social exclusion and highlight the central role of TPB's elements (Ajzen, 1991; Baker, 2006; Goffman, 1971; Sandikci and Holt, 1998) (e.g. subjective norms, perceived behavioural control etc.) in connection with channel use; they also confirm the direct applicability of TPB for the examined theoretical issues and pave the way for its future use. More importantly, we have a similar set of findings for all channels involved in relation to social exclusion. A major finding is that increased levels of social exclusion could result in generating increased confidence towards shopping; the latter presents an invaluable finding for policy makers who could capitalise on this by developing subsequent policy measures.

Social exclusion has a negative effect on attitudes towards shopping online by using a computer. The relationship is not statistically significant for shopping at the mall or for shopping online via a 
mobile phone. This presents another unique finding showing clear differences for the role of social exclusion in relation to attitudes for the three channels under analysis.

In contrast, perceived behavioural control and subjective norms have a positive effect on attitudes towards shopping via all channels. Therefore, these findings denote the positive contribution of two TPB dimensions towards attitude formation and extend relevant work which examined human behaviour issues in the context of similar online and offline channels (Carrington et al., 2014; Hsu et al., 2006; Pookulangara et al., 2011). Social exclusion has a positive effect on intentions to shop online using a mobile phone. The relationship was not significant for shopping online using a computer and shopping at the mall. This is an original finding showing the distinctive role of the mobile channel towards intentions to shop online. Perceived behavioural control and attitude towards shopping via a channel have a positive effect on intentions to shop via the respective channel. Subjective norms also have a positive effect on intentions to shop via a mobile phone. However, this relationship was negative for shopping online via a computer and not statistically significant for shopping at the mall. These findings provide novel insights for the link between social exclusion and shopping via mobile phones and, therefore, they provide a contribution to the relevant, contemporary literature (see for example Basel and Gips, 2014). Hence, our work stresses the distinctive role of a specific channel (mobile phones) when examining the social exclusion and wellbeing phenomena and, surprisingly, contrasting findings were generated for the other two channels. More importantly, this finding can support future policy making especially when mobile phones present an ideal channel to target socially excluded consumers. Overall, we argue that the mobile channel can be an important channel for boosting consumer access to products and, in turn, it can increase product consumption too especially for socially excluded consumers.

Social exclusion has a positive effect on the perceived utilitarian value of shopping online via a computer. However, the relationship is not statistically significant for the mobile phone and the traditional shopping channel. This is an important finding as we will normally expect utilitarian value to be associated with most online shopping channels; however, in the case of socially excluded consumers, the optimum online shopping channel is the computer. Attitude towards shopping via a 
specific channel has a positive effect on the perceived utilitarian value of online shopping via a computer or a mobile phone. The relationship was not statistically significant for shopping at the mall. In addition, perceived behavioural control, subjective norms, and intentions also have a positive effect on the perceived utilitarian value that the respective channel offers to the respondents. These findings provide an insightful comparison for the role of various channels and social exclusion in relation to perceived utilitarian value when shopping via these channels. They also illustrate the synergistic and interrelated role of various behavioural elements (perceived behavioural control, subjective norms, intentions) towards the creation of utilitarian value. More importantly, a specific channel seems to enjoy an increasing role (e.g. computer) for the examined issues which, as far as we know, represents an original finding and makes a large contribution to relevant academic work (Babin et al., 1994; Bellenger et al., 1977; Chitturi et al., 2008; Oppewal et al., 2013; Reynolds et al., 2012). This finding also indicates that computers represent the most "consumer-friendly" channel to compare, contrast and evaluate product offerings and, hence, it supports the maximisation of utilitarian value of socially excluded consumers. This could be related to the fact that computers provide an easy and relaxed platform for shopping for these consumers whilst shopping in the mall can be stressful and time consuming and mobile shopping cannot be ideal for prolonged, numerous comparisons, evaluations of prices and product ordering; this will be extremely useful for future policy making too considering the heightened role of computers for socially excluded consumers.

The degree to which respondents consider themselves as socially excluded has a positive effect on the perceived hedonic value experienced via shopping online via a computer and via shopping at the mall. In contrast, this relationship is not statistically significant when shopping online via a mobile phone. Subjective norms, attitude and intentions to shop via a specific channel also have a positive effect on the perceived hedonic value experienced when shopping via the respective channel. However, the effect of the perceived behavioural control on the hedonic value is negative for all channels. These findings illustrate a plethora of similarities and differences between channels and stress the role of each channel in relation to specific behavioural elements. The perceived utilitarian value experienced when shopping via a specific channel has a positive effect on the hedonic value experienced when shopping 
via the respective channel. These findings generate interesting insights for the role of various channels and social exclusion in relation to perceived hedonic value when shopping via these channels (especially via the computer and the mall). A major association is also established for the positive role of perceived utilitarian value on the hedonic value experienced when shopping via the same channel. The latter presents an outstanding theoretical contribution to the current literature (see for example Babin et al., 1994; Bellenger et al., 1977; Chitturi et al., 2008; Oppewal et al., 2013; Reynolds et al., 2012) and merits further research.

Social exclusion also has a positive effect on the perceived contribution of all channels on respondents' wellbeing. In contrast, perceived behavioural control has a negative effect on the perceived contribution of online shopping using a computer on respondents' wellbeing. The relationship is not statistically significant for the other two channels. Subjective norms have a positive effect on the perceived contribution of all channels to wellbeing. Attitude towards shopping online using a mobile phone has a negative effect on the perceived contribution of this channel to wellbeing. The relationship between attitude towards the remaining two channels and the contribution of the respective channel to respondents' wellbeing is not statistically significant; these findings highlight the different types of effect in connection with wellbeing.

Intentions towards shopping online using a mobile phone also have a positive effect. The perceived utilitarian value that this channel offers positively influences the contribution of the channel to wellbeing. Finally, the hedonic value that all channels offer has a positive influence on respondents' wellbeing. The above findings offer a plethora of novel insights for the role of channels towards wellbeing and extend past research into the role of traditional channels (Dennis et al., 2007; Hedhli et al., 2013) and the smart online ones (Fiore et al., 2005; Konus et al., 2008).

\section{Conclusion and future research}

"Shopping is an activity through which the self finds expression in, and becomes subjected to, the situations at hand." (Prus and Dawson, 1991) In this paper we have examined the effect of social exclusion on the retail channel preferences as manifested through the psychological process leading to 
intention of use and by examining the perceived value gain we studied the impact on the consumer's well-being. A key contribution emanating from this work is that people who consider themselves to be socially excluded have greater intentions to shop via a mobile phone and those intentions lead to greater channel contribution to wellbeing, relationships that are not apparent for the other two channels. People who are socially excluded consider that accessing products and services by mobile phone plays an important role in their quality of life in the community, and their social and leisure wellbeing. These important benefits of shopping via a mobile phone for people who are socially excluded hold for disabled as well as able and rural as well as urban residents. The benefits even hold for the financiallydistressed as well as those who are not financially distressed although financial distress is associated with lower wellbeing. The benefits also hold for older people as well as younger ones although older age is associated with lower wellbeing. Shopping via mobile phone therefore has an important part to play in improving the wellbeing of socially-excluded people, such as the old, disabled and even those with financial troubles. The latter is a distinctive contribution to the computers in human behaviour literature as it highlights the wider, far reaching application of our work, which draws attention to benefits in accessing products and services via mobile phones for a range of categories of socially excluded people. This addresses the need for further contribution in the theoretical underpinning (Burton, 2005; Wensley, 1995); the previous work which has examined the relationship between retailers and their customers (Walsh et al., 2016) as well as the factors which influence adoption of innovation by individuals (Foxall, 1994). Despite the above, we need to stress that computers may still be the preferred platform for socially excluded consumers who seek to maximise utilitarian value via shopping as it offers relevant benefits to these consumers.

Our work has generated numerous findings that will be beneficial to managers and practitioners. For example, the contrasting role of three channels and their resultant contribution towards wellbeing were noted and, therefore, managers and practitioners are advised to consider these issues when devising strategies targeting socially excluded people. The increasing role of mobile phones was also stressed in this work, and this has become a major shopping channel on its own right. Therefore, mobile phones could be ideal devices to approach socially excluded people, especially as our work has shown 
the resultant wellbeing benefits associated with their use. More importantly, this work has indicated the "universal" influence of shopping via a mobile phone for all categories of socially excluded consumers (e.g. disabled, financially distressed, older etc.). Managers need to factor this in when devising appropriate strategies and campaigns aiming to maximise accessibility to products and services.

Finally, this work has shown that consumers can perceive utilitarian value when shopping and that this utilitarian value can positively affect their perceptions of hedonic value. This positive influence of utilitarian value on hedonic value holds for each of three channels, computer, mobile and mall shopping. The results indicate that affective marketing communications (that build hedonic value) should be more effective than cognitive marketing communications (that build utilitarian value) in boosting shoppers' wellbeing. Nevertheless, cognitive marketing communications should have an important part to play not only in building utilitarian value but also hedonic value. Managers and practitioners should therefore aim to design communications at the point-of-sale that not only build hedonic value, but also utilitarian value. Hedonic value can be built by applying, for example, attractive or entertaining video (via digital signage in the case of the mall channel (Dennis et al., 2014)) or 3D (Alharabat and Dennis, 2010) or virtual reality (VR) (Papagiannidis et al., 2013; Papagiannidis et al. 2017) presentation (online). The effectiveness can be boosted by integrating cognitive textual information such as product/service and performance details into the video, 3D or VR point-of-sale presentation (Algharabat and Dennis, 2010; Dennis et al., 2014). Managers could also consider how media multitasking affects users as this has been show to encourage impulse buying (Chang 2017).

This work can be extended in a number of ways in the future. Firstly a longitudinal study could shed light on how the social exclusion manifests over time and at different times of the year (e.g. during holiday and festival seasons). Similarly the dimensions of social exclusion can be decomposed and measured in more detail. These two changes in the research design could help overcome the limitation of treating social exclusion as a homogenous time invariant construct and potentially offer useful practical insights for different types of retailers. Future research could also examine how marketers can play a wider role in their customers' wellbeing by interacting with them in more appropriate ways, perhaps giving a very different meaning to the term "retail therapy". For instance, shopping assistants' 
training could go beyond training the assistants on the products and services on offer and include consumer psychology sessions. Online, data analytics and personal information collected could help identify patterns that could be used to personalise the design of online stores and mobile apps. Such innovations could help not only deliver a better customer experience, but lead to closer relationship and, in turn, loyalty. Finally, it would be of interest to have a more culturally and contextually varied sample in order to examine how different cultures (either measured on a personal or national level) influence retail channel preferences and perceived value gain. For instance it may be of interest to explore how culture dimensions (Hofstede and Bond, 1984) such as individualism vs collectivism might moderate the relationships in our model.

\section{References}

Ajzen I. (1991) The theory of planned behavior. Organizational Behavior and Human Decision Processes 50(2): 179-211.

Algharabat R and Dennis C (2010) 'Using Authentic 3D Product visualisation for an electrical online retailer', Journal of Customer Behaviour, 9(2): 97-116.

Babin BJ, Darden WR and Griffin MD. (1994) Work and/or fun: measuring hedonic and shopping value. Journal of Consumer Research 20(644-656).

Baker SM. (2006) Consumer normalcy: Understanding the value of shopping through narratives of consumers with visual impairments. Journal of Retailing 82(1): 37-50.

Basel A and Gips J. (2014) Tablets, touchscreens, and touchpads: How varying touch interfaces trigger psychological ownership and endowment. Journal of Consumer Psychology 24(2): 226-233.

Belk R. (2015) Youtube on the couch: psychoanalytic challenges in a digital age. Marketing Theory 15(1): 21-24.

Bellenger DN, Robertson DH and Greenberg BA. (1977) Shopping Center Patronage Motives. Journal of Retailing 53(Summer): 29-38.

Blázquez, M. (2014) Fashion shopping in multichannel retail: The role of technology in enhancing the customer experience. International Journal of Electronic Commerce 18: 97-116.

Burchardt T, Le Grand J and Piachaud D. (1999) Social exclusion in Britain 1991-1995. Social Policy and Administration 33(3): 227-244.

Burton D. (2005) Marketing theory matters. British Journal of Management 16: 5-18.

Carrington MJ, Neville BA and Whitwell GJ. (2014) Lost in translation: Exploring the ethical consumer intention-behavior gap. Journal of Business Research 67: 2759-2767.

Chang, Y. The influence of media multitasking on the impulse to buy: A moderated mediation model, Computers in Human Behavior, Volume 70, May 2017, Pages 60-66.

Chitturi R, Raghunathan R and Mahajan V. (2008) Delight by design: The role of hedonic versus utilitarian benefits. Journal of Marketing 72(3): 48-63.

Calvo-Porral, C., \& Levy-Mangin, J.-P. (2015). Switching behavior and customer satisfaction in mobile services: analyzing virtual and traditional operators. Computers in Human Behavior, 49, 532540.

Dennis, C., Alamanos, E., Papagiannidis, S and Bourlakis, M. (2015) Does social exclusion influence multiple channel use? The interconnections with community, happiness and wellbeing', Journal of Business Research 69(3): 1061-1070. 
Dennis C, Brakus J, Gupta S and Alamanos E (2014) 'The effect of digital signage on shoppers' behavior: The role of the evoked experience', Journal of Business Research, 67(11): 2250-2257

Dennis C, King T, Wright LT, et al. (2007) A Commentary on Social and Experiential (e-)Retailing. International Journal of Retail and Distribution Management 35(6): 443-456.

Field A. (2005) Discovering Statistics Using SPSS, London: Sage Publications.

Fiore AM, Jin HJ and Kim J. (2005) For fun and profit: Hedonic value from image interactivity and responses toward an online store. Psychology and Marketing 22 (8): 669-694.

Foxall GR. (1994) Consumer initiators: Adaptors and innovators. British Journal of Management 5: S3S12.

Goffman E. (1971) Relations in Public: Microstudies of the Public Order., New York: Basic Books.

Gao, L., Waechter, K. A., \& Bai, X. (2015). Understanding consumers' continuance intention towards mobile purchase: a theoretical framework and empirical study- A case of China. Computers in Human Behaviour, 53, 249-262.

Guy CM. (1998) Controlling new retail spaces: the impress of planning policies in Western Europe. Urban Studies 35(8): 953-979.

Hair JF, Black WC, Babin BJ, et al. (2010) Multivariate data analysis. Global perspective, USA: Pearson.

Harris L and Dennis C. (2011) Engaging customers on Facebook: Challenges for e-retailers. Journal of Consumer Behaviour 10 (6): 338-346.

Hedhli KE, Chebat JC and Sirgy MJ. (2013) Shopping well-being at the mall: Construct, antecedents, and consequences. Journal of Business Research 66 (7): 856-863.

Hewer P and Campbell C. (1997) Appendix: Research on Shopping - A Brief History and Selected Literature. The Shopping Experience. SAGE Publications Ltd. London: SAGE Publications Ltd, 186-207.

Hill RP. (2008) Disadvantaged consumers: An ethical approach to consumption by the poor. Journal of Business Ethics 80 (1): 77-83.

Hofstede G and Bond MH. (1984) Hofstede's Culture Dimensions. Journal of Cross-Cultural Psychology 15(4): 417-433.

Hsu MH, Yen CH, Chiu CM, et al. (2006) A longitudinal investigation of continues online shopping behaviour: An extension of the theory of planned behaviour. International Journal of Human - Computer Studies 64: 889 - 904.

Huxley P, Evans S, Madge S, et al. (2012) Development of a social inclusion index to capture subjective and objective life domains (Phase II): psychometric development study. Health Technology Assessment 16(1): 1-241.

Irvine A. (2016) Social, Economic and Health Impacts of WaveLength's Work with Loneliness and Isolation. Available at: http://www.york.ac.uk/inst/spru/research/pdf/WaveLength.pdf.

Kang, J.-Y. M., Mun, J. M., \& Johnson, K. K. P. (2015). In-store mobile usage: downloading and usage intention toward mobile location-based retail apps. Computers in Human Behaviour, 46, 210217.

Kim Y-K, Kang J and Kim M. (2005) The relationships among family and social interaction, loneliness, mall shopping motivation, and mall spending of older consumers. Psychology and Marketing 22(12): 995-1015.

Konus U, Verhoef PC and Neslin SA. (2008) Multichannel Shopper Segments and Their Covariates. Journal of Retailing 84 (4): 398-413.

Liu C and Forsythe S. (2011) Examining drivers of online purchase intensity: Moderating role of adoption duration in sustaining post-adoption online shopping. Journal of Retailing and Consumer Services 18(1): 101-109.

Marketline. (2013) Online retail in the United States. Available at: http://advantage.marketline.com.proxy.library.lincoln.ac.uk/Product?pid=MLIP1062-0010.

Monsuwe TP, Dellaert BGC and Ruyter K. (2004) What drives consumers to shop online? A literature review. International Journal of Service Industry Management 15(1): 102. 
Oppewal H, Tojib DR and Louvieris P. (2013) Experimental analysis of consumer channel-mix use. Journal of Business Research 66(11): 2226-2233.

Pantano, E., and Priporas, C.-V. (2016). Mobile retailing effect on consumption experiences: A dynamic perspective. Computers in Human Behavior, 61: 548-555.

Pantano, E. and Timmermans, H. (2014) What is smart for retailing?, Procedia Environmental Sciences 22: 101-107.

Papagiannidis, S., Pantano, E., See-to, E., \& Bourlakis, M. (2013). Modelling the determinants of a simulated experience in a virtual retail store and users' product purchasing intentions. Journal of Marketing Management.

Papagiannidis S, Pantano E, See-To E, Dennis C and Bourlakis M (2017earlycite) 'To immerse or not? Experimenting with two virtual retail environments', Information Technology \& People, 30(1).

Peace R. (2001) Social Exclusion: A Concept in Need of Definition Social Policy. Journal of New Zealand 16(July): 17-35.

Pew Internet. (2012) Teens Fact Sheet, . Available at: http://www.pewinternet.org/fact-sheets/teensfact-sheet/.

Piacentini M, Hibbert S and Al-Dajani H. (2001) Diversity in deprivation: exploring the grocery shopping behaviour of disadvantaged consumers. The International Review of Retail, Distribution and Consumer Research 11(2): 141-158.

Pookulangara S, Hawley J and Xiao G. (2011) Explaining consumers' channel-switching behavior using the theory of planned behavior. Journal of Retailing and Consumer Services 18(4): 311-321.

Prawitz AD, Garman ET, Sorhaindo B, et al. (2006) In charge financial distress / financial well-being scale: Development, administration, and score interpretation. Financial Counseling and Planning 17(1): 34-50.

Prus R and Dawson L. (1991) Shop 'til You Drop: Shopping as Recreational and Laborious Activity. The Canadian Journal of Sociology / Cahiers canadiens de sociologie 16(2): 145-164.

Reynolds KE, Jones MA, Findley Musgrove C, et al. (2012) An investigation of retail outcomes comparing two types of browsers. Journal of Business Research 65(8): 1090-1095.

Sandikci O and Holt DB. (1998) Malling society: mall consumption practices and the future of public space. ServiceScapes: The concept of place in contemporary markets: 305-336.

Stanley JK, Hensher DA, Stanley JR, et al. (2011) Mobility, social exclusion, and well-being: Exploring the links. Transportation Research Part A 45 (8): 789-801.

Swaine B, Labbe D, Poldma T, et al. (2014) Exploring the Facilitators and Barriers to Shopping Mall Use by Persons with Disabilities and Strategies for Improvements: Perspectives from Persons with Disabilities, Rehabilitation Professionals and Shopkeepers. European Journal of Disability Research 8: 217-229.

Tauber EM. (1972) Why do people shop? Journal of Marketing 36 (4): 46-49.

Taylor MP, Jenkins SP and Sacker A. (2011) Financial capability and psychological health. Journal of Economic Psychology 32: 710-723.

Walsh G, Albrecht AK, Kunz W, and Hofacker CF. (2016) Relationship between online retailers' reputation and product returns. British Journal of Management 27: 3-20.

Wei TT, Marthandan G, Chong AYL, et al. (2009) What drives Malaysian m-commerce adoption? An empirical analysis. Industrial Management \& Data Systems 109 (3): 370-388.

Wensley R. (1995) A critical review of research in marketing. British Journal of Management 6 (S1): S63-S82.

Westlake T. (1993) The disadvantaged consumer: problems and policies. In: Bromley R and Thomas CJ (eds) Retail Change: Contemporary Issues. London: UCL Press.

Williams P and Hubbard P. (2001) Who is disadvantaged? Retail change and social exclusion. The International Review of Retail, Distribution and Consumer Research 11(3): 267-286.

Wrigley N, Guy C and Lowe M. (2002) Urban regeneration, social inclusion and large store development: The seacroft development in context. Urban Studies 39 (11): 2101-2114. 
Yang K. (2012) Consumer technology traits in determining mobile shopping adoption: An application of the extended theory of planned behaviour. Journal of Retailing and Consumer Services 19: 484-491.

Zinkhan GM. (2005) The marketplace, emerging technology and marketing theory. Marketing Theory 5(1): 105-115. 